\title{
Phytochemical and Spectral Studies of Synthesis Sulfur Nanoparticles Using Sophora japonica Pods Extract
}

\author{
Akl M. Awwad ${ }^{1^{*}}$, Nidá M. Salem ${ }^{2}$, Amany O. Abdeen ${ }^{1}$ \\ ${ }^{1}$ Royal Scientific Society, El Hassan Science City, P.O. Box 1438 Amman 11941 Jordan \\ Akl.awwad@yahoo.com \\ ${ }^{2}$ Plant Protection Department, Faculty of Agriculture, the University of Jordan, Amman, Jordan \\ nmsalem72@gmail.com \\ ${ }^{1}$ Royal Scientific Society, El Hassan Science City, P.O. Box 1438 Amman 11941 Jordan \\ Amany.abdeen@rss.jo
}

\begin{abstract}
This study aims to investigate the aqueous extract of Sophora japonica pods for the presence of various phytochemicals and to synthesize sulfur nanoparticles. The presence of various phytochemicals viz. polyphenols, alkaloids, terpenoids, flavonoids, and tannins were investigated by standard biochemical methods. A rapid, green and novel approach for synthes is sulfur nanoparticles (SNPs) from sodium thiosulfate in the presence of Sophora japonica pods aqueous extract in one-pot reaction at ambient temperature. The resulting sulfur nanoparticles were characterized by Fourier transform infrared spectroscopy (FT-IR), X-ray diffraction (XRD) and scanning electron microscopy (SEM). The results show that sulfur nanoparticles were successfully synthesized in sphere shape, and with an average particle size 5-100 nm. The effect of plant pods extract concentration on particle size of sulfur nanoparticles shows that can significantly reduce the particle size without changing the shape. The results revealed that the aqueous extract of Sophora japonica pods act as capping, dispersing and stabilizing agent for sulfur nanoparticles. This method is a novel approach for production nanosized sulfur particles, which could be applied to prepare sulfur nanoparticles for application in antimicrobial activity, fertilizers, and plant protection.
\end{abstract}

Keywords: Phytochemical synthesis; sulfur nanoparticles; Sophora japonica pods extract; characterization
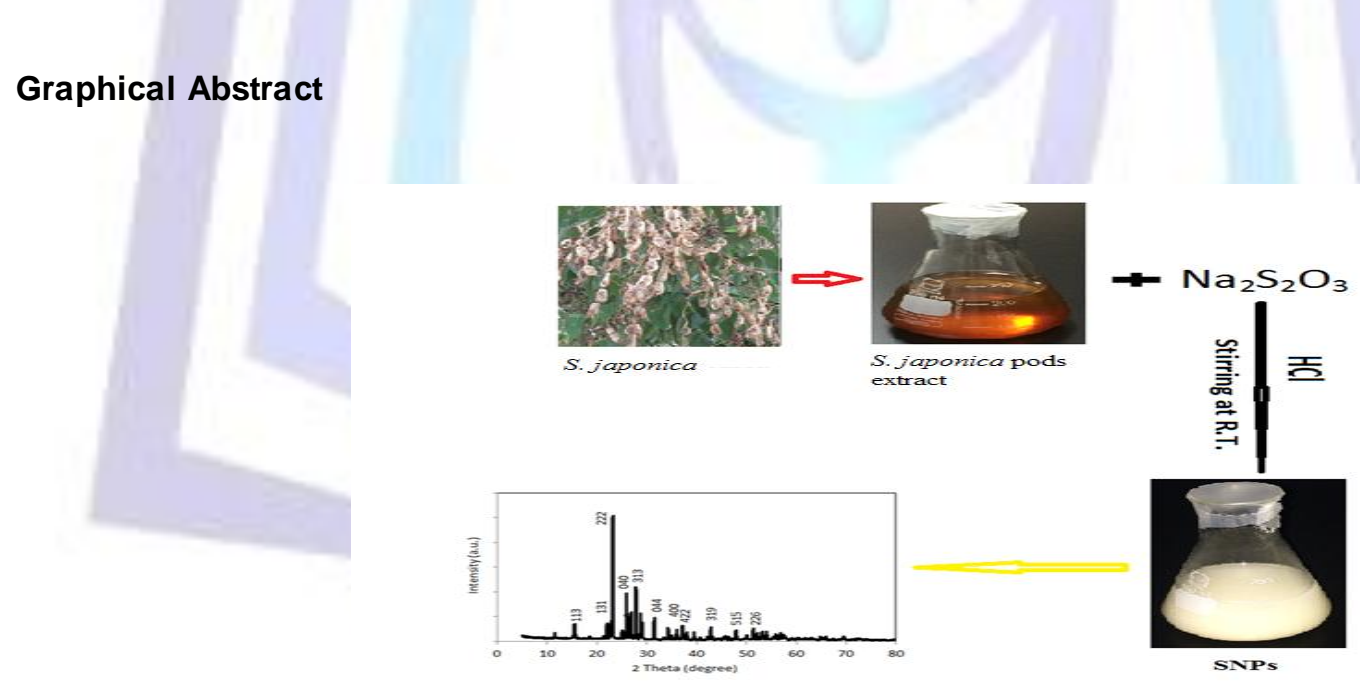

\section{Council for Innovative Research}

Peer Review Research Publishing System

\section{Journal: Journal of Advances in Chemistry}

\author{
Vol. 11, No.3 \\ editorjaconline@gmail.com
}




\section{INTRODUCTION}

Sulfur is widely used in different applications such as fertilizers, phamaceuticals, rubber and fibres industries, bioleaching processes, manufacturing sulfuric acid, antimicrobial agents, insecticides and fumigants, etc. It is a common fungicide for grapes, strawberry, many vegetables and several other crops and has a good efficiency against a wide range of powdery mildew diseases as well as black spot. Despite the practicable applications of sulfur, limited research works are available in the open literature related to synthesis of sulfur nanoparticles. Synthesis of sulfur nanoparticles (SNPs) have been achieved using various routes including microemulsion method [1, 2], surfactant assisted route [3, 4], an electrochemical method [5, 6], Eggshell membrane as natural biomaterial [7], supersaturated method in the presence of organic modifiers polyethylene glycol (PEG 600) and triethanolamine [8], sublimed sulfur in in a green solvent polyethylene glycol-200 [9], precipitation method [10], from $\mathrm{H}_{2} \mathrm{~S}$ gas by using biodegradable iron chelate catalyst in reverse micro emulsion technique [11], and polysulfide decomposition with various organic and inorganic acids [12]. These methods have many disadvantages due to the difficulty of scale up of the process, separation and purification of nanoparticles from the microemulsion (oil, surfactant, co-surfactant and aqueous phase, and consuming huge amount of surfactant. In this study, we report for the first time a novel, rapid, cost-effective and environmentally biosynthesis of sulfur nanoparticles using Sophora japonica pods extract, which act as capping, dispersing and stabilizing agent.

Sophora japonica is medium sized tree grows to 65 feet in height, usually with a broad round crown. The 6 to 10 inch, bright-green, pinnate leaves are fern-like and consist of 9 to 15, elliptic leaflets. The leaves are extremely pest-free. The tree begins to bloom when 10 to 15 years old. In late summer and early fall, 10 -inch to 15 -inch upright panicles of mildly fragrant, creamy-white, pea-like flowers are produced at the ends of branches and live about a month. Flowers are replaced by ornamental yellow seed pods, 6 to 8 inches long, which persist well into the winter and resemble strings of beads. Fig 1 shows a photograph of Sophora japonica tree and its pods.

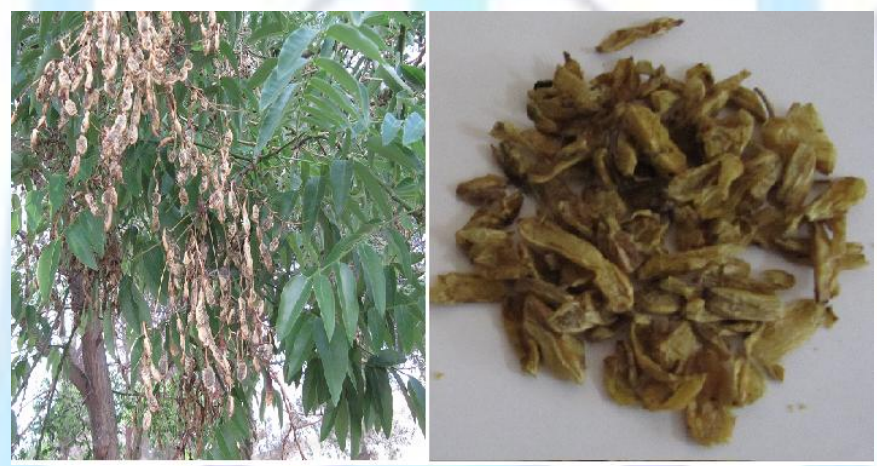

Fig. 1. Photograph of Sophora japonica tree and its pods

\section{EXPERIMENTAL}

\subsection{Materials}

Sodium thiosulfate, $\mathrm{Na}_{2} \mathrm{~S}_{2} \mathrm{O}_{3}$ and hydrochloric acid, $\mathrm{HCl}$ were obtained from E-Merck. All chemicals were used as received without any further purification. Sterile distilled water was used with condu ctivity $1 \mu \mathrm{S} / \mathrm{cm}$.

\subsection{Preparation of Sophora japonica pods extract}

Pods were collected from Sophora japonica trees planted at the campus of Royal Scientific Society, El Hassan Science City, Amman, Jordan. Pods were washed several times with distilled water to remove the dust particles and then sun dried to remove the residual moisture. The dried pods were grounded to a fine powder in a grinding mill (Retsch RM 100, Thermo Fisher Scientific, NH, USA) and sieved to get size fraction < $44 \mu \mathrm{m}$. $20 \mathrm{gram}$ of Sophora japonica pods ( $S j p$ ) powder was boiled in $500 \mathrm{ml}$ glass beaker along with $400 \mathrm{ml}$ of sterile distilled water for 10 minutes. After boiling, reddish-brown colour solution is formed and allowed to cool at room temperature. The aqueous extract of pods was separated by filtration with Whatman No.1 filter paper and then centrifuged at $1200 \mathrm{rpm}$ for 5 minutes to remove heavy biomaterials. The aqueous extract was stored at room temperature to be used for green synthesis of sulfur nanoparticles.

\subsection{Synthe sis of sulfur nanoparticles}

In a typical reaction synthesis, sulfur nanoparticles (SNPs) synthesized as follows: an appropriate amount of so dium thiosulfate, $\mathrm{Na}_{2} \mathrm{~S}_{2} \mathrm{O}_{3}$ was dissolved in $50 \mathrm{ml}$ of Sophora japonica pods (Sjp) extract under mild stirring for 5 minutes at room temperature and then diluted to $100 \mathrm{ml}$ by sterile distilled water. Afterwards hydrochloric acid was added with rate $1 \mathrm{ml} / \mathrm{min}$ with mild stirring for allowing the sulfur precipitations uniformly. The suspended sulfur particles obtained were separated by centrifugation at $1000 \mathrm{rpm} / \mathrm{min}$ for 5 minutes and then repeatedly washed with sterile distilled water to remove any biological materials. The sulfur nanoparticles are divided into two parts. In the first part, the sulfur nanoparticles are remained in the sterile distilled water without any additives as prepared. In the second part, the sulfur nanoparticles after purification were dried in a vacuum at $80^{\circ} \mathrm{C}$ for $6 \mathrm{~h}$. The product was light yellow powder used for SEM, XRD, and FTIR analysis. 


\subsection{Phytochemical screening of Sophora japonica pods aque ous extract.}

Major bioactive constituents in sophora japonica pods extract was determined qualitatively using the standard methods [13-15]. Bioactive materials analysis revealed the presence of alkaloids, flavonoids, steroids, tannins,, and phenolic compounds.

\subsection{Characterization techniques}

Crystalline sulfur nanoparticles were examined by X-ray diffractometer (XRD-6000 Shimadzu, Japan) equipped with $\mathrm{Cu} \mathrm{Ka}$ radiation source using $\mathrm{Ni}$ as filter and at a setting of $30 \mathrm{kV} / 30 \mathrm{~mA}$. All XRD data were collected under the same experimental conditions, in the angular range $3^{\circ} \leq 2 \theta \leq 50^{\circ}$. FTIR Spectra for Sophora japonica seed extract and sulfur nanoparticles were obtained in the range $4000-400 \mathrm{~cm}^{-1}$ by IR-Prestige-21, FTIR spectrophotometer (Shimaduz, Japan) using KBr pellet method. Scanning electron microscopy (SEM) analysis of sulfur nanoparticles was done using Quanta FEI 450 SEM machine.

\section{RESULTS AND DISCUSSION}

The XRD analysis of sulfur nanoparticles synthesized is illustrated in Fig.2. The $2 \theta$ peaks at $15.4^{\circ}, 21.98$, $23.08^{\circ}, 25.8^{\circ}, 27.76^{\circ}, 31.45^{\circ}, 34.22^{\circ} 37.02^{\circ}, 42.08^{\circ}, 47.62^{\circ}, 50.02^{\circ}$ are attributed to the crys tal planes of sulphur at $113,131,222,040,313,044,400,422,319,515$, and 226, respectively. The sulfur nanoparticles are wellcrystalline and the position and the relative intensity of the diffraction peaks match well with the standard monoclinic phase sulfur diffraction pattern (JCPDS N-34-094). The average particle sizes of the synthesized sulfur nanon particles were calculated using Debye-Scherrer formula [16]:

$\mathrm{D}=\mathrm{K} N / \beta \cos \theta$

Where $D$ is the mean diameter of nanoparticles, $\beta$ is the full width at half-maximum value of XRD diffraction lines, $\lambda$ is the wavelength of $X$-ray radiation source $0.15405 \mathrm{~nm}, \theta$ is the half diffraction angle-Bragg angle and $\mathrm{K}$ is the Scherrer constant with value from 0.9 to 1 . The unassigned peaks in XRD pattern are thought to be related to crystalline and amorphous organic phases of Sophora japonica pods extract. The crystalline size of sulphur nanoparticles calculated from Scherrer equation iwas about $60 \mathrm{~nm}$.
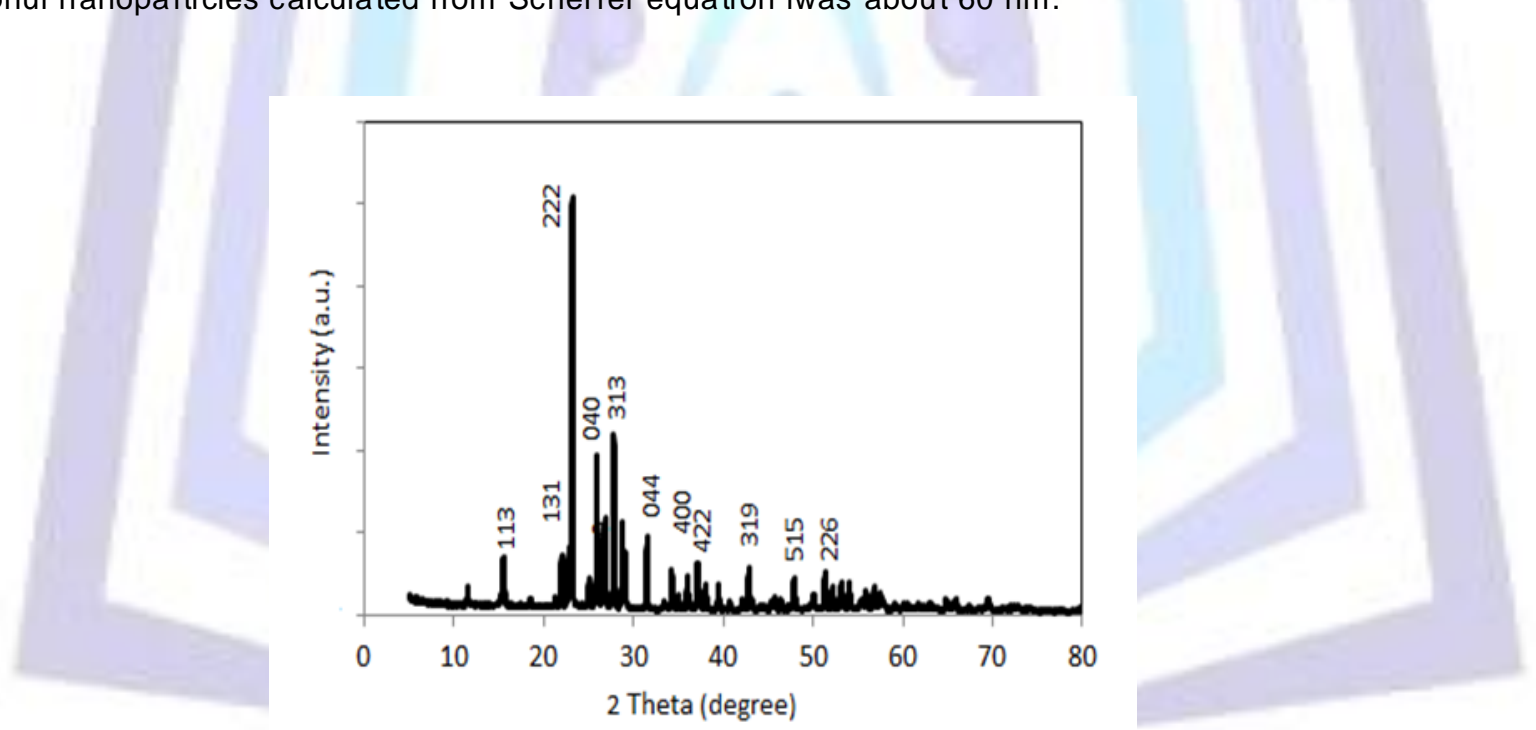

Fig.2. XRD pattern of synthesized sulfur nanoparticles

FT-IR analysis was carried out to identify the possible biomolecules responsible for the capping and stabilization of sulfur nanoparticles synthesized by Sophora japonica pods extract. The powder extracts display a number of absorption peaks, reflecting their complex nature. FT-IR spectrum of Sophora japonica pods extract is shown in Fig.2. FT-IR spectrum of Sophora japonica pods extract display strong absorption bands at $3433 \mathrm{~cm}^{-1} \mathrm{could}_{\text {be }}$ ascribed to the stretching absorption band of amino $(-\mathrm{NH})$ and hydroxyl $(-\mathrm{OH})$ stretching $\mathrm{H}$-bonded alcohols and phenols. The strong absorption peaks at $2912 \mathrm{~cm}^{-1}$ and $2845 \mathrm{~cm}^{-1}$ could be assigned to the as ymmetric and symmetric stretching of $-\mathrm{CH},-\mathrm{CH}_{2}$ and $-\mathrm{CH}_{3}$ functional groups of aliphatic. The bands at $1651 \mathrm{~cm}{ }^{-1}$ is characteristic of amide carbonyl group in amide I and amide II. The amide band I assigned to the stretch mode of the carbonyl group coupled to the amide linkage while the amide II band arises as a result of the $\mathrm{N}-\mathrm{H}$ stretching modes of vibration in the amide linkage. The peak at $1508 \mathrm{~cm}^{-1}$ could be attributed to $\mathrm{N}-\mathrm{O}$ stretching in nitro compounds. The band at $1431 \mathrm{~cm}^{-1}$ is assigned to the methylene scissoring vibrations from the proteins. C-N stretch of aromatic amines and carboxylic acids gives rise to bands at $1357 \mathrm{~cm}^{-1}$. The peak at $1253 \mathrm{~cm}^{-1} \mathrm{can}$ be due to C-O vibrations of alcohols, phenols and C-N stretching vibrations of amine. The band at $1041 \mathrm{~cm}^{-1}$ 
assigned to the $\mathrm{C}-\mathrm{O}$ stretching vibrations of alcohols $\mathrm{C}-\mathrm{N}$ stretching vibrations of amine. Additional peaks at 883 $\mathrm{cm}^{-1}$ and $559 \mathrm{~cm}^{-1}$ can be assigned to bending modes of aromatic compounds.

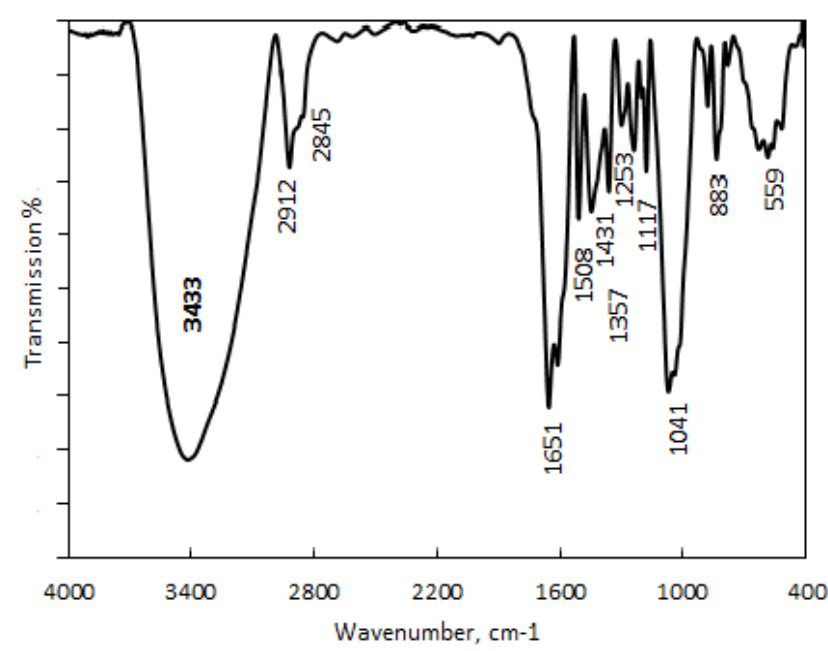

Fig. 3. FT-IR spectra of Sophora japonica pods extract.

Fig. 4 and 5 show the FT-IR spectra of the synthesized sulfur nanoparticles. FT-IR spectra of SNPs indicate a new chemistry linkage on the surface of sulfur nanoparticles. This suggests that Sophora japonica pods extract can bind to sulfur nanoparticles through carbonyl of the amino acid residues in the protein of the extracts, therefore acting as stabilizer and dispersing agent for synthesized sulfur nanoparticles and prevent agglomeration of sulfur nanoparticles. All the characteristic peaks of Sophora japonica pods extract were observed in FT-IR spectra of sulphur nanoparticles. No significant new peak appeared, indicating there was no chemical reaction but physical cross -linking between pods extracts and sulphur nanoparticles. The FT-IR spectra of the sulfur nanoparticles show a strong and sharp peak at $467 \mathrm{~cm}^{-1}$.

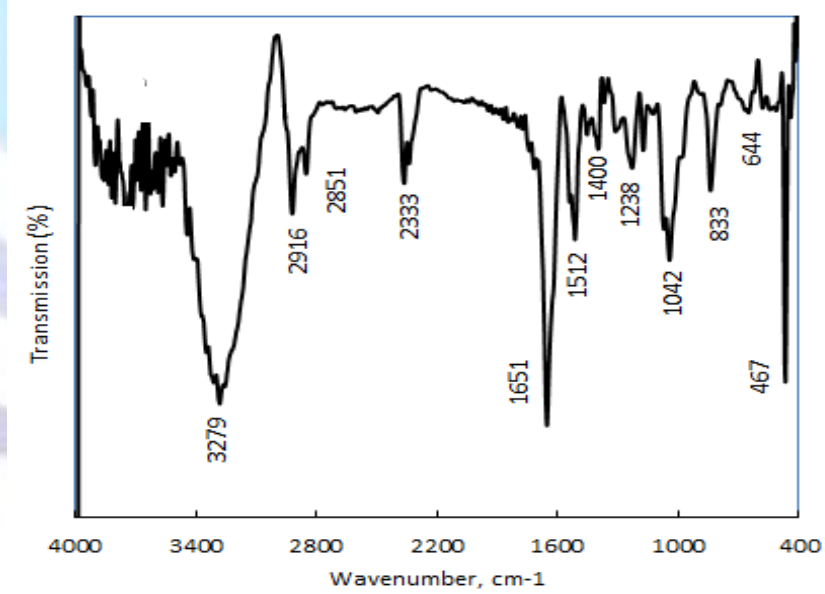

Fig. 4. FT-IR of Sjp-sulfur nanoparticles. 


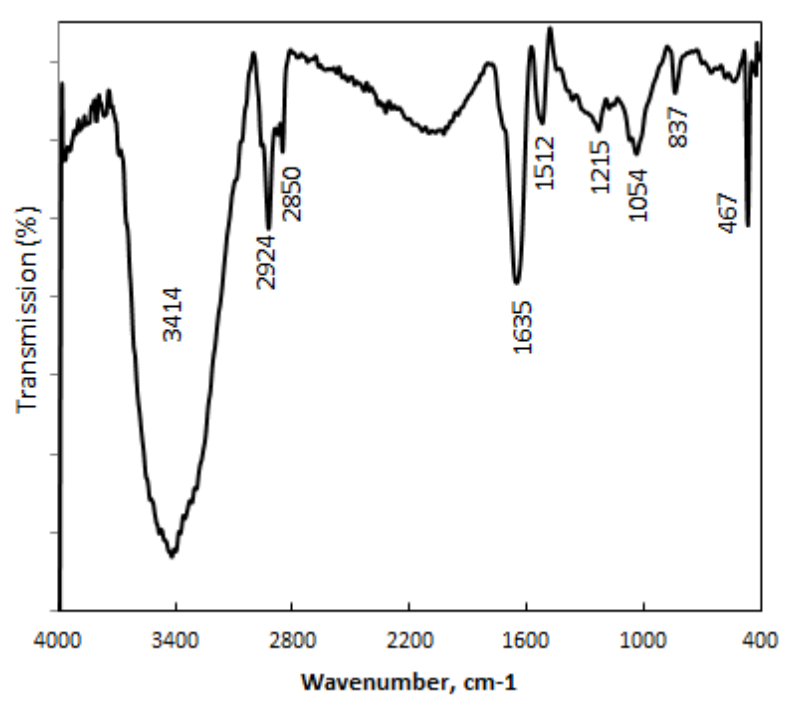

Fig. 5. FT-IR spectra of purified synthesized sulphur nanoparticles

The results of the phytochemical analysis of Sophora japonica pods aqueous extract showed that the terpenoids, flavonoids, alkaloids, tannins, amino acids, and phenols were found to be present in afore mentioned pods extract. The plant pods screened for phytochemical constituents seemed to have the potential to act as a source of capping and als o to stabilize the nanoparticles as a result of the presence of various compounds that are vital for dispersing, capping, and stabilization.

The suspended sulfur nanoparticles in sterile distilled water were used for scanning electron microscopy, SEM by fabricating a drop of suspension onto a clean electric stubs and allowing water to completely evaporate. The SEM images of the sulfur nanoparticles synthesized by Sophoria japonica pods extract, SEM image, Fig. 6 shows all the particles are homogeneous ly dispers ed and ranges approximately from $5-80 \mathrm{~nm}$. The shape of the sulfur nanoparticles is spherical with few exceptional as ellipsoidal. The larger sulfur nanoparticles may be due to the aggregation of the smaller ones, due to the SEM measurements. It was found the increasing the amount of plant extract in the reaction mixture leads to decrease the particles size of sulfur nanoparticles and their agglomeration tendencies. Moreover, Sophora japonica pods extract produce the smallest particles.

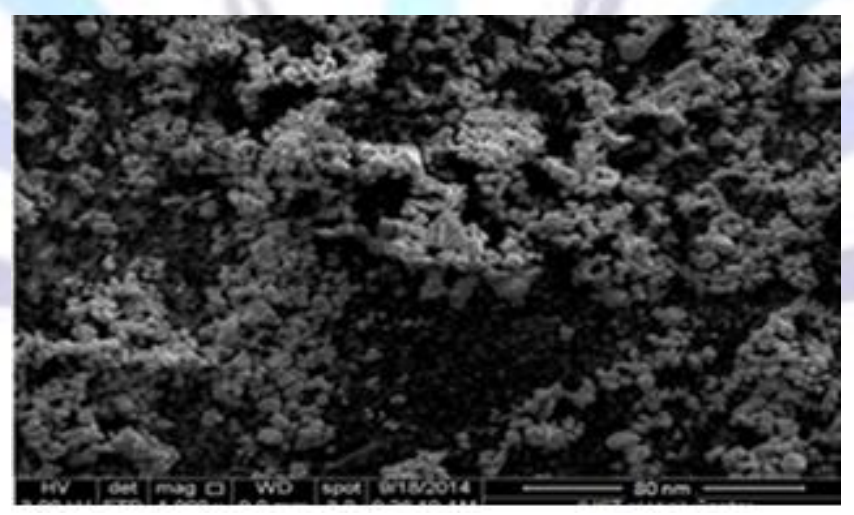

Fig. 6. SEM image of synthesized sulphur nanoparticles 


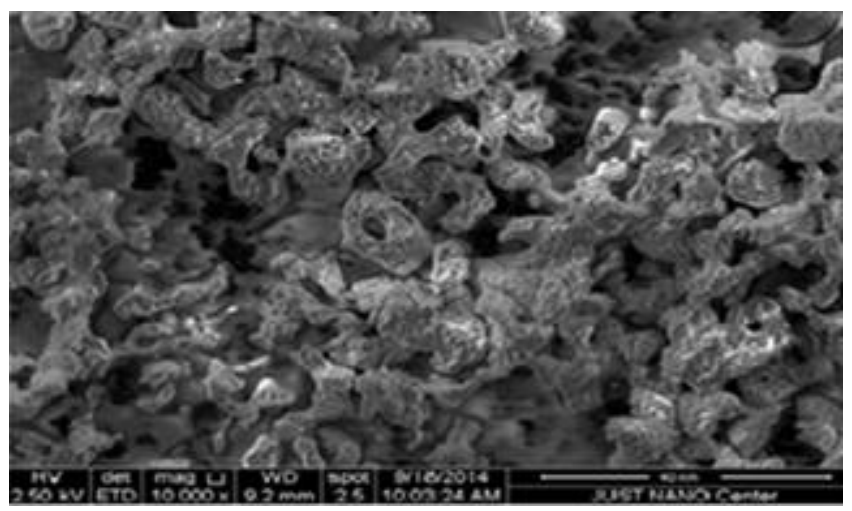

Fig.7. SEM images of synthesized sulphur nanoparticles dried at $80^{\circ} \mathrm{C}$ for $6 \mathrm{~h}$.

The SEM micrograph in Fig. 7 demonstrates that, during sulfur nanoparticles drying at high temperatures and the spherelike shape of the particles persists, even though it is a nonequilibrium shape for SNPs. At high temperature, the spherical break into isometric particles.

An initial phase sodium thiosulfate was dissolved and mixed with sophora japonica spods extract. Hydrochloric acid was then added to the solution which releases sulfur in the solution, which dispersed and capped by $S$. japonica pods extract. The reaction is described as follows:

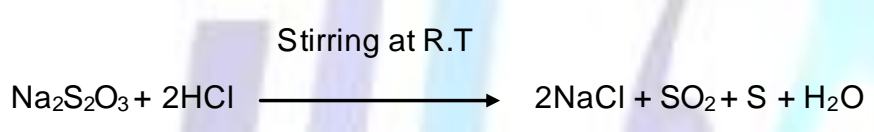

S.japonica pods extract

The following scheme represents the mechanism behind the formation of sulfur nanoparticles

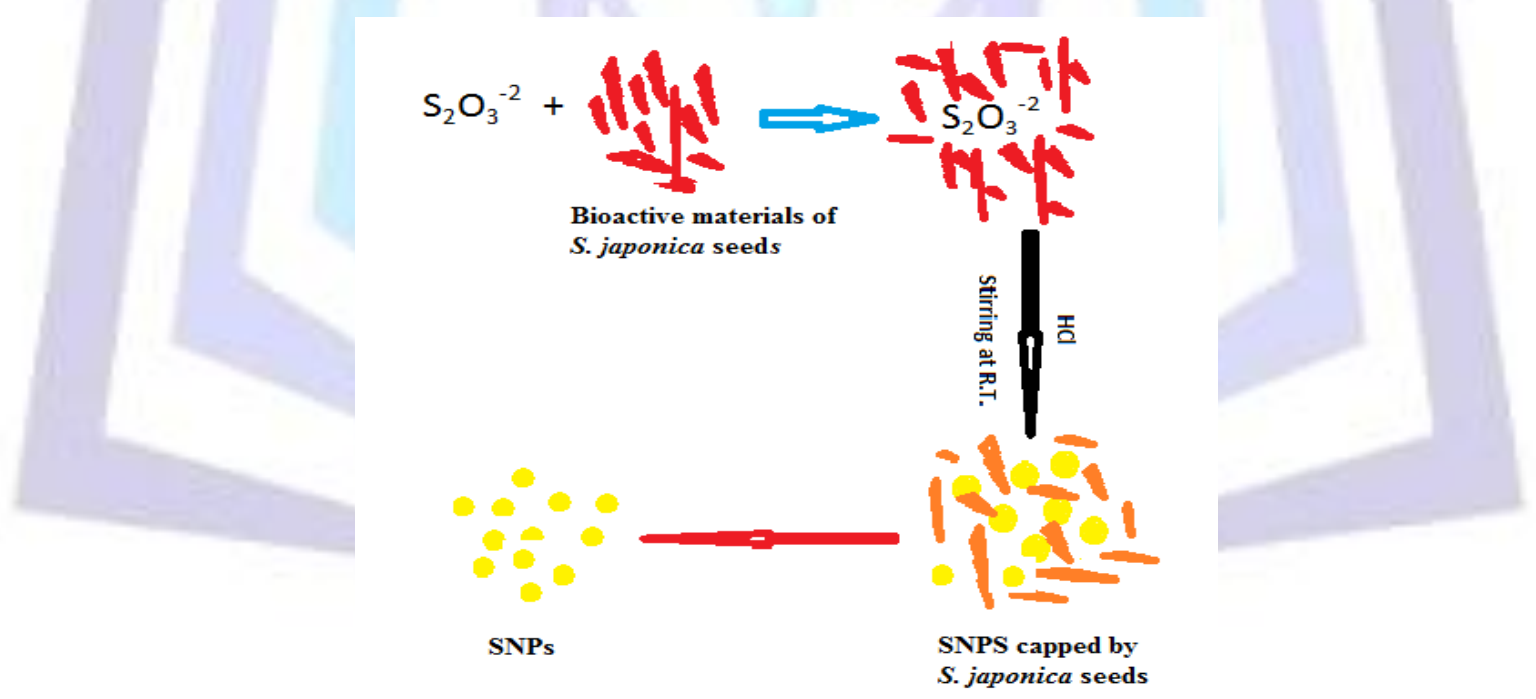

\section{CONCLUSIONS}

A novel approach for green synthesis of sulfur nanoparticles by using Sophora japonica pods extract in one-pot liquid phase reaction at ambient temperature and atmospheric pressure has been reported for first time. The described method gives highly crystalline pure sulfur nanoparticles with unifom shape and average particle size of 5-100 nm. The concentration of plant pods extract and sodium thiosulfate ions are playing an important role in the green synthesis of sulfur nanoparticles. XRD, SEM and FT-IR support the formation and stability of the biosynthesized SNPs. The sulfur nanoparticles had an antibacterial activity against bacterial pathogens. Thus it can be concluded that plant pods extract can be used as simple, low-cost and environmentally friendly biomaterial acting as stabilizing and dispersing agent in synthes is of SNPs with antibacterial activity. 


\section{ACKNOWLEDGMENTS}

This work was supported by Grant No. 47/2014, Abdul Hameed Shoman Fund for Support of Scientific Research, Jordan. Authors also are grateful to Royal Scientific Society and the University of Jordan for providing all facilities to carry out this research work.

\section{REFERNCES}

[1] Guo, Y., Zhao, J., Yang, S., Yu, K., Wang, Z., Zhang, H. Preparation and characterization of monoclinic sulfur nanoparticles by water-in-oil microemulsions technique. Powder Technology, 162 (2006) 83-86.

[2] Soleimani, M., Aflatouni, F., Khani, A. A new and simple method for sulfur nanoparticles synthesis. Colloid Joumal, 75 (2013) 112-116.

[3] Chaudhuri, R.G., Paria, S.J. Synthes is of sulfur nanoparticles in aqueous surfactant solutions. Colloid and Interface Sci., 343 (2010) 439-446.

[4] Chaudhuri, R.G., Paria, S.J. Growth kinetics of sulfur nanoparticles in aqueous surfactant solutions. J. Colloid and Interface Sci., 354 (2011) 563-569.

[5] Shamsipur, M., Poourmortazavi S, M., Roushani , M., Kohsar, I., Hajimirsadeghi, S. Novel approach for electrochemical preparation of sulfur nanoparticles. Microchimica Acta, 173 (2011) 445-451.

[6] Chen, J-J., Jia X., She q-J., Wang, C., Zhang, Q., Zheng, M-s., Dong, Q-f. The preparation of nanosulfur/MWCNTs and its electrochemical performance. Electrochimica Acta, 55 (2010) 8062-8066.

[7] Cheng , X., Cheng, K., Liu, J., Sun, X. Synthesis and characterization of nanoparticles sulfur using eggshell membrane as template. Mater. Sci. Forum, 675-677 (2011) 279-282.

[8] Wu, H., Wang, A., Yin, H., Zhang, D., Jiang, T., Zhang, R., Liu, Y. preparation of sulfur sheets by supersaturated solvent method in the presence of organic modifiers. Mater. Lett., 62 (2008) 1996-1998.

[9] Xie X-y, Li L-y, Zheng P-s, Zheng W -j, Bai Y, Cheng T-f, Liu A. Facile synthesis, spectral properties and formation of sulfur nanorods in PEG-200. J Mater Res Bulletin 2012; 47: 3665-3669.

[10] Guo, Y-M., Deng, Y-H., Zhao, J-Z., Wang, Z-C., Zhang, H-B. Synthesis and characterization of sulfur nanoparticles by liquid phase precipitation method. Acta Chim.Sinica, 63 (2005) 337-341.

[11] Deshpande, A.S., Khomane, R.B., Vaidya, B.K., Joshi, R.M., Harie, A.S., Kulkarni, B.D. Sulfur nanoparticles synthesis and characterization from $\mathrm{H}_{2} \mathrm{~S}$ gas using novel biodegrable iron chelates in W/O microemulsion. Nanoscale Res. Lett., 3 (2008) 221-229.

[12] Massalimov, I.A.S., Shainurova, A.R., Khusainov, A.N., Mustafin, A.G. Production of sulfur nanoparticles from aqueous solution of potassium polysulfide. Russian J. Appl. Chem., 85 (2012) 1832-1837.

[13] Harborne, J.B. Phytochemical methods, London Chapman and Hall, Ltd, 1973.

[14] Hussain, I., Khattak, M.U.R., ullah, R., Muhammad, Z., Khan, N., Khan, F.A., Ullah, Z., Haider, S. Phytochemicals screening and antimicrobial activities of selected medicinal plants of Khyberpakhtunkhwa Pakistan. African J. Pharm Phamacology, 5 (2011) 746-750.

[15] lqbal, P.J. Phytochemical screening of certain plant species of agracity. J. Drug Delivery \& Therapeutics 2 (2012) 135-138.

[16] Klug, H.P., Alexander, L.E. X-ray diffraction procedure for polycrystalline and amorphous materials Wiley, New York, 1954.

\section{Authoirs' information}

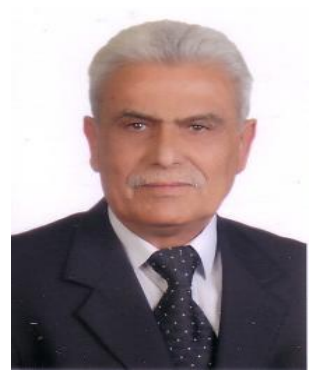

Akl M. Awwad is professor and senior scientist at Royal Scientific Society, El Hassan Science City, Amman, Jordan. He received his Ph.D. degree from the department of pure and applied chemistry, Strathclyde University, Glasgow, U.K. He has published over 100 peer reviewed articles, 10 books and holds 7 patents. His current research interests include the green synthesis of the advanced Nano materials, chemical thermodynamics and water treatment.

Nidá M. Salem is associate professor at Faculty of Agriculture, the University of Jordan, Amman, Jordan.

Amany O. Abdeen is assistant researcher at Royal Scientific Society. . 